\title{
Efficacious Approach to Integrating Health and Wellness Coaching into the Clinical Care for Weight Management: A Systematic Review and Proposal
}

\author{
Jenny Seunghyun Lee ${ }^{1,2,3^{*}}$, Sarah Alpini ${ }^{4}$
}

${ }^{1}$ Assistant Professor, Department of Family Medicine (Patient Care Center \#215), Texas College of Osteopathic Medicine, University of North Texas Health Science Center, 3500 Camp Bowie Blvd, Fort Worth, TX, USA

${ }^{2}$ Founder \& Director, The Goodness Holistic Wellness and Lifestyle Medicine Program (From Gene to Community; from Community to Gene), Texas College of Osteopathic Medicine, University of North Texas Health Science Center, 3500 Camp Bowie Blvd, Fort Worth, TX, USA

${ }^{3}$ Director, Community Engagement in Pain Research, Texas College of Osteopathic Medicine, University of North Texas Health Science Center, 3500 Camp Bowie Blvd, Fort Worth, TX, USA

${ }^{4} 2^{\text {nd }}$ Year Medical Student, Texas College of Osteopathic Medicine, University of North Texas Health Science Center, 3500 Camp Bowie Blvd, Fort Worth, TX, USA

*Corresponding author: Jenny Seunghyun Lee, Family Medicine, Texas College of Osteopathic Medicine University of North Texas Health Science Center, 3500 Camp Bowie Blvd, Fort Worth, TX 76107-2699; Tel: 817-735-0521;

E-mail: jenny.lee@unthsc.edu

\begin{abstract}
Objectives: Current clinical obesity management is insufficient in helping patients initiate and sustain weight loss, requiring innovative and efficacious interventions. We investigated health and wellness coaching in clinical care to motivate patients to adopt healthier lifestyles towards weight management, and proposed an efficacious approach to integrating coaching into clinical care for weight management.

Methods: We conducted a systematic review of literature for the past 10 years concerning health and wellness coaching for weight loss in a primary care setting. Relevant articles were organized by the approach that coaching was applied towards weight management. Studies were considered efficacious if the coaching intervention resulted in clinically meaningful weight loss ( $>5 \%$ initial body weight in six months) or was statistically significant compared to control/standard care.

Results: Health and wellness coaching in a variety of clinical settings at moderate-to-high frequency dosages was most efficacious in helping patients achieve and sustain clinically meaningful weight loss, as seen in nine of the thirteen studies examined. Other results of coaching included improved diabetes and cardiovascular risk factors, healthy lifestyle behaviors, and self-sufficiency for maintaining these changes.

Conclusions: Health and wellness coaching incorporated into clinical care results in positive health outcomes. It is recommended for clinicians to integrate coaching into the clinical care of obese and overweight patients. We propose coaching for weight management should be delivered at least a moderate frequency $(>1 /$ month) by a trained health and wellness coach. Further, coaching can be personalized to SUIT patient needs and optimize care.
\end{abstract}

Received date: November 09, 2016

Accepted date: December 16, 2016

Published date: December 21, 2016

Citation: Lee, S.J., et al. Efficacious Approach to Integrating Health and Wellness Coaching into the Clinical Care for Weight Management: A Systematic Review and Proposal. (2016) J Diab Obes 3(3): 1 - 9.

DOI: $10.15436 / 2376-0494.16 .1209$

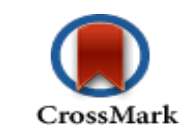




\section{Introduction}

Obesity is a global disease affecting nearly forty percent of adults worldwide ${ }^{[1]}$. In United States over two-thirds of adults are obese or overweight ${ }^{[2-8]}$,while $17 \%$ of children and adolescents considered obese to date ${ }^{[3-9]}$. Years of research outline the vast, growing morbidities and mortality associated with obesity, including an increased risk of diabetes ${ }^{[7,10-15]}$, cardiovascular disease ${ }^{[2,11-16]}$, and cancer ${ }^{[2,11,15]}$. Obesity and overweight are also associated with decreased longevity ${ }^{[7,14,17,18]}$. In addition to physiological consequences, obesity and overweight are associated with a decreased quality of life ${ }^{[3,11]}$, psychosocial adversities $^{[3,11,19,20]}$, decreased wages ${ }^{[19]}$ and a reduced probability of employment $^{[3,11,16,19]}$. Obese and overweight individuals commonly experience prejudice, discrimination, social stigma, anxiety, depression, low self-esteem, and bullying ${ }^{[11-21]}$. Obesity is a complex disease that develops due to an interaction between lifestyle, genetic predisposition, and environmental interaction ${ }^{[13]}$. The dietary patterns seen in obesity and overweight also typically overlap with those that contribute to the weight gain and endocrine dysfunction, which may result in diabetes ${ }^{[10,21]}$. Obesity is also a well-documented economic burden, driving health care costs annually ${ }^{[1,15,22,23]}$, largely due to the co-morbidity-related complications of obesity ${ }^{[1,23]}$.

Despite a multitude of research showing the adverse affects of obesity on every organ system, the prevalence of obesity is continuing to increase. The global prevalence of overweight and obesity for adults has increased from $28.8 \%$ in 1980 to $36.9 \%$ in 2013 for men and from $29.8 \%$ to $38.0 \%$ for wom$\mathrm{en}^{[24]}$, suggesting current obesity prevention and management is inadequate. The US Preventative Services Task Force (USPSTF) recommends physicians screen all adult patients for obesity ${ }^{[8]}$. Patients with a BMI $>25 \mathrm{~kg} / \mathrm{m}^{2}$ are classified as overweight, while a patient with a BMI of $30 \mathrm{~kg} / \mathrm{m}^{2}$ or greater is considered obese and should be referred or offered intensive multi component behavioral interventions ${ }^{[8]}$. Other researchers agree, stating primary care physicians should prescribe and treat overweight and obese patients with moderate-intensity (at least monthly) lifestyle interventions ${ }^{[25]}$. According to the American Academy of Pediatrics (AAP), children and adolescents between two and 18 years of age should also be screened annually via their gender-specific BMI ${ }^{[26]}$. Children with a BMI between the $5^{\text {th }}$ and $85^{\text {th }}$ percentile are considered a healthy weight, while children below the $5^{\text {th }}$ percentile are underweight. A BMI $>85^{\text {th }}$ to $94^{\text {th }}$ percentile classifies children and adolescents as overweight, while at $\mathrm{BMI}>95^{\text {th }}$ percentile pediatric patients are considered obese. The USPSTF recommends behavioral interventions for overweight and obese children ages 6 and older in order to achieve an improved weight status ${ }^{[26]}$.

Experts agree that the best treatment for obesity and overweight is prevention of the disease altogether ${ }^{[9,16,22]}$. This role seems to fall to PCPs who are responsible for guiding their patients in best health practices, including diet and exercise recommendations. PCPs familiar with a patient's social history can tailor a prevention or treatment plan according to their patient's socioeconomic status, culture, or childhood developmental stage $^{[22]}$. This tailored care often includes referring patients to other health care team members. For example, physical therapists can help patients increase mobility and exercise tolerance, dieticians can teach patients about healthier eating habits, and social workers can assist patients to address financial barriers to healthy lifestyles. These health care professionals working with PCPs highlight the importance of a patient-centered team approach to obesity and overweight management as well as general healthcare. Yet, literature has shown discrepancies that exist as to how often physicians counsel patients for weight-loss, diet, and exercise ${ }^{[27-29]}$, although patients are more motivated to engage in weight-loss behavior following their Physician's advice to do $\mathrm{so}^{[19,22]}$. Further, many physicians report they lack the confidence and skills to help patients lose weight ${ }^{[11,18]}$, despite vast medical training and clinical experience in health management. Physicians report barriers to communication about obesity to include their fear of offending their patient, limited patient interest and motivation, personal lack of training and resources, and a small likelihood of success ${ }^{[9,11,12,18,30]}$. Others may include a lack of reimbursement for efforts to promote weight loss ${ }^{[22]}$ and cost of care ${ }^{[31]}$. Another major hurdle is limited visit time ${ }^{[32]}$, especially when PCPs may need to consider acutely fatal diseases ahead of obesity in a span of a fifteen minute visit ${ }^{[33]}$.

Concerning the need for sustainable, healthy weight outcomes, the best management for obesity is prevention ${ }^{[9,16,22]}$. However, experts agree the best treatment for patients who are obese or overweight is intensive behavioral lifestyle modification $^{[4,22]}$, which can be accomplished through health and wellness coaching. Health and wellness coaching is a patient-centered partnership designed to empower patients towards achieving healthier lifestyles. Coaches facilitate the patients in self-assessment, generating solutions to meet goals, and developing the skills and confidence to become responsible for improving their lifestyles ${ }^{[34,35]}$. The initial session is an introduction to the coaching process to orient the client. Subsequent sessions involve the coach discussing with the client about the progress they are making towards previous goals, barriers they have encountered, setting new goals based on the discussion, and scheduling the next appointment ${ }^{[36]}$. The coach maintains a dialogue by using open-ended statements, and encouraging the patient to explore solutions to meet their goals ${ }^{[36]}$. The coach guides the client in setting goals and developing a plan centered on their values, sense of purpose, and inspiration to change ${ }^{[26,29]}$. The client is steered by the coach to self-assess their current status and move towards self-realization of steps they can make to achieve their goals. The coach also ensures the client is setting "SMART" (Smart, Measurable, Action-oriented, Realistic, and Time-bound ${ }^{[35,37,38]}$ goals. The coach does this by encouraging the client, linking their goals to their values and vision of future self, and helping them stay accountable ${ }^{[38]}$.

Health and wellness coaching is successful because the client develops the skills, mindset, and autonomy necessary to pursue lifelong wellness changes ${ }^{[29,31-39]}$. Effective, sustainable behavior change in the client occurs because the coaching process generates internal motivation centered on what is important to the client ${ }^{[37]}$. This internal motivation develops through a recurring cycle of empathy by the coach for barriers faced by patient's barriers to change, discerning personal motivation for change, assessing and building confidence towards making steps to change, setting SMART goals, and creating a plan for accountability in these changes ${ }^{[34]}$. Client share accountability with the coach for these changes, as they are responsible for generating solutions according to what is motivating them. This differs from the "expert" approach adapted by many time-con- 
strained physicians who use their knowledge and expertise to treat, educate, answer questions for, and advise their patients in a more disease-oriented fashion ${ }^{[29,37,39]}$. While this expert approach is efficacious for diagnosing and treating physiological diseases, it may not provide the necessary stimulus to promote psychological changes such as lifestyle behaviors. Researchers suggest this may be because people respond unreliably to expert advice via compliance or defiance, while the collaboration in coaching encourages the patient to problem-solve instead ${ }^{[37]}$. The coaching process is also longitudinal. Clients work with coaches on a regular basis for an extended time, which enables them to make slow and steady progress towards change and develop a lasting relationship with their coach.

The growing literature has shown that the coaching process reduces the risk of chronic disease by promoting behavior change ${ }^{[29,35,40,41]}$. Significant changes in psychological, behavioral, physiological, and social outcomes have resulted following coaching in adults with chronic disease ${ }^{[42]}$. Coaching also resulted in improvement in quality of life, depressive symptoms, and stress levels with maintenance of these changes at follow-up ${ }^{[20]}$, and has been used to help physicians facing burnout or dissatisfaction with their practice ${ }^{[39]}$. Further, coaching has been useful to support behavior changes toward weight loss, by helping patients become motived to start and maintain exercise regimens, eat healthier, and make healthier lifestyle choices ${ }^{[11]}$. Others report improvements in health measures including exercise adherence $^{[35,40]}$, mood and mental health ${ }^{[40,43]}, \mathrm{HbA} \mathrm{C}^{[35,40,41,43]}$, fasting glucose $^{[40]}$, blood pressure ${ }^{[35,40]}$, body mass index ${ }^{[40]}$, resting heart rate $^{[40]}$, body weight ${ }^{[8]}$, and waist circumference ${ }^{[43]}$. Coaching is also useful to support behavior change and weight loss in people with serious mental illnesses, improving their self-regulation for exercise and exercise self-efficacy, decreasing mood disturbances, improving fasting glucose, blood pressure, and resting heart rate $^{[28]}$. The positive results of coaching in clinical care studies implicate a need to integrate coaching into clinical practice for weight loss intervention.

However, the question remains: how do physicians integrate health and wellness coaching into their practice to effectively help overweight and obese patients achieve and maintain meaningful weight loss? What modalities of the coaching process have been adopted and implemented in a clinical practice to increase its efficacy? To this end, we investigated: 1) Current trends in clinical obesity and overweight management; 2) Coaching outcomes for obese and overweight patients; and 3) The methods and process of implementing coaching into a clinical practice.

\section{Methods}

We conducted a literature review of the application of health and wellness coaching intervention in clinical practice for weight management. An English-language literature search of the databases PubMed, Scopus, Academic Search Complete, Google Scholar, and Science Direct was conducted from May to August 2016 and literature older than ten year (published before 2006) was excluded. The search terms used were 'wellness coaching', 'lifestyle coaching', 'health coaching', 'wellness coaching AND obesity', 'physician perception AND wellness coaching', 'addressing obesity AND physician', 'overweight AND wellness coaching', 'physician AND diagnosing obesity', 'overweight AND wellness coaching', 'addressing obesity in the primary care setting', 'physician perception + body image + overweight'.

Abstracts were reviewed for relevance and studies investigating wellness, lifestyle, or health coaching in the context of non-obesity were excluded. This included persons with mental health diseases, pregnant women, other means of behavioral modification, and more. Interventions utilizing pharmacologic agents were beyond the scope of this review and therefore excluded. Additional publications were also obtained from searching bibliographies of cited references.

Selected studies were analyzed according to the approach that coaching was applied towards weight management including: who was administering health and wellness coaching, how patients received health and wellness coaching, dosage of health and wellness coaching treatment by length and frequency of sessions, and the results of treatment. Studies were considered efficacious if weight loss was clinically meaningful ( $>5 \%$ initial body weight) or the weight loss intervention was significant compared to control/standard care treatment.

\section{Results}

Literature extensively shows health and wellness coaching is efficacious for weight management in a variety of primary care settings for obese and overweight patients ranging from children to adults (Table 1). Coaching has been successfully implemented in the care of children ${ }^{[44,45]}$, adolescents ${ }^{[46]}$, and adults ${ }^{[10,47-52]}$. Coaching has also been incorporated into patient care with a variety of health professionals. This has been achieved with coaches plus PCP collaboration ${ }^{[8,10,4244,53]}$, with coaches alone ${ }^{[31,44-50,52,53]}$, with physician coaches ${ }^{[34,46]}$, and with auxiliary health professional coaches such as Medical Assis$\operatorname{tants}^{[38,47,54]}$, Nurse Practitioners ${ }^{[50]}$, and Registered Dietician Nutritionists $^{[31]}$.

As to a method of implementing coaching in a clinical care process, this has been done for groups of patients ${ }^{[10]}$, parent-child groups ${ }^{[44,45]}$, via one-on-one sessions ${ }^{[10,31,34,38,45-50,52]}$. Coaching was also integrated in-person ${ }^{[10,31,34,46-51,54]}$ and remotely via telephone ${ }^{[10,45,49,52]}$, a study-specific website ${ }^{[10]}$, email support $^{[10,14]}$, as well as general mail, text message, and virtual video visit $^{[45]}$.

The dosage of coaching interventions offered to patients varied as well including low-intensity or less than monthly coaching ${ }^{[31,4547,50]}$, moderate-intensity or at least monthly coaching $^{[10,31,4951,54]}$, high-intensity or at least weekly ${ }^{[10,34,44,46-52]}$, or with a combination of these throughout the intervention ${ }^{[10,31,47]}$. Length of intervention ranged from approximately four months ${ }^{[34,44,46]}$, six months ${ }^{[47,52,54]}$, approximately one year ${ }^{[45,48-50]}$, and multiple years $^{[10,31,51]}$.

Concerning health improvements, coaching has helped patients achieve clinically meaningful weight $\operatorname{loss}^{[10,31,47-51,54]}$, maintain significant weight loss for two years ${ }^{[10,51]}$, decrease waist circumference ${ }^{[50]}$, decrease blood presure from hypertensive to normo tensive ${ }^{[49]}$, improve BMI from obese to normal[ ${ }^{[38,41,48]}$, normalize blood glucose levels ${ }^{[31,48]}$, and normalize cholesterol levels ${ }^{[31]}$. Other benefits of coaching included a general weight and fat $\operatorname{loss}^{[14,44,52,54]}$, lowered blood pressure ${ }^{[46]}$, lower triglyceride levels ${ }^{[46]}$, improved $\mathrm{BMI}^{[43-45]}$, lower HbAlc levels ${ }^{[43]}$, improved anthropomorphic body measurements ${ }^{[46]}$, 
and behaviors conducive to weight $\operatorname{loss}^{[14,44,45,52]}$.

TABLE 1: Results of Health and Wellness Coaching in Clinical Care Practice for Weight Management.

\begin{tabular}{|c|c|c|c|c|c|c|c|}
\hline Author & Design & Coach & Subjects & Intervention & Coaching Dose & Control & Outcomes \\
\hline $\begin{array}{l}\text { Appel et } \\
\text { al. }{ }^{[10]}\end{array}$ & $\begin{array}{l}\text { RCT; HWC } \\
\text { vs. single } \\
\text { counseling } \\
\text { session }\end{array}$ & $\begin{array}{l}\text { Health } \\
\text { Coaches }\end{array}$ & $\begin{array}{l}415 \\
\text { obese } \\
\text { adult } \\
\text { patients } \\
\text { with at } \\
\text { least } 1 \\
\text { CV risk } \\
\text { factor }\end{array}$ & $\begin{array}{l}\text { In-person intervention } \\
\text { included one-on-one or } \\
\text { group HWC, access to } \\
\text { study-specific website, } \\
\& P C P \text { collaboration } \\
\text { Remote intervention in- } \\
\text { cluded telephonic HWC } \\
\text { along with telephonic, } \\
\text { email, and Internet } \\
\text { support }\end{array}$ & $\begin{array}{l}\text { Two-year in- } \\
\text { tervention with } \\
\text { weekly HWC } \\
\text { during first } \\
\text { three months, } \\
\text { monthly HWC } \\
\text { during next } \\
\text { three months, } \\
\text { and twice } \\
\text { monthly HWC } \\
\text { for remainder } \\
\text { of study (18 } \\
\text { months) }\end{array}$ & $\begin{array}{l}\text { Brief in- } \\
\text { tervention } \\
\text { coun- } \\
\text { seling } \\
\text { at study } \\
\text { onset }\end{array}$ & $\begin{array}{l}\text { Clinically meaningful weight } \\
\text { loss achieved and sustained in } \\
\text { both intervention groups, with } \\
\text { weight loss for both interven- } \\
\text { tion groups being statistically } \\
\text { significant compared to control } \\
(\mathrm{P}<0.001) \text { with more individu- } \\
\text { als in the in-person HWC group } \\
\text { achieving } 5 \% \text { or more weight } \\
\text { loss }(\mathrm{P}=0.73) \text { compared to } \\
\text { remote HWC }\end{array}$ \\
\hline $\begin{array}{l}\text { Ball et } \\
\text { al. }{ }^{[46]}\end{array}$ & $\begin{array}{l}\text { RCT; Com- } \\
\text { paring HWC } \\
\text { to single } \\
\text { counseling } \\
\text { session }\end{array}$ & $\begin{array}{l}\text { Physi- } \\
\text { cians with } \\
\text { expertise in } \\
\text { nutrition, } \\
\text { exercise, } \\
\text { and/or men- } \\
\text { tal health }\end{array}$ & $\begin{array}{l}46 \text { obese } \\
\text { adoles- } \\
\text { cents, } \\
\text { ages } \\
13-17\end{array}$ & $\begin{array}{l}\text { HIP: One-on-one HWC } \\
\text { for education, self-mon- } \\
\text { itoring, goal change, } \\
\text { behavior and cognitive } \\
\text { change, addressing } \\
\text { barriers to change, and } \\
\text { setting lifestyle goals } \\
\text { aimed at increasing } \\
\text { healthy behaviors } \\
\text { YLP: one-on-one HWC } \\
\text { for education, self-mon- } \\
\text { itoring, goal setting, } \\
\text { and setting lifestyle } \\
\text { behaviors goals aimed } \\
\text { at reducing unhealthy } \\
\text { behaviors }\end{array}$ & $\begin{array}{l}16 \text { weekly, one- } \\
\text { on-one, } 45-60 \\
\text { minute HWC } \\
\text { sessions for a } \\
\text { span of four } \\
\text { months }\end{array}$ & $\begin{array}{l}\text { One } \\
\text { coun- } \\
\text { seling } \\
\text { session } \\
\text { and edu- } \\
\text { cational } \\
\text { maternal }\end{array}$ & $\begin{array}{l}\text { HIP HWC group had the greatest } \\
\text { improvements in } \mathrm{BMI}(\mathrm{P}= \\
0.002) \text { and weight }(\mathrm{P}=0.004) \\
\text { compared to control, although } \\
\text { differences between HIP and } \\
\text { YLP were insignificant. } \\
\text { Compared to control, HWC } \\
\text { groups had greater weight loss } \\
(\mathrm{P}=0.004 \text { for HIP, } \mathrm{P}=0.025 \\
\text { for YLP) and decreased BMI ( } \mathrm{P} \\
=0.0002 \text { for HIP, } \mathrm{P}=0.03 \text { for } \\
\text { YLP). }\end{array}$ \\
\hline $\begin{array}{l}\text { Frates et } \\
\text { al. }{ }^{[34]}\end{array}$ & Case Study & $\begin{array}{l}\text { Physi- } \\
\text { cian with } \\
\text { Lifestyle } \\
\text { Medicine } \\
\text { and HWC } \\
\text { training }\end{array}$ & $\begin{array}{l}\text { Adult, } \\
\text { over- } \\
\text { weight } \\
\text { female }\end{array}$ & $\begin{array}{l}\text { Patient administered } \\
\text { therapeutic walking } \\
\text { sessions with one-on- } \\
\text { one coach and used } \\
\text { time to discuss, reflect, } \\
\text { and set goals }\end{array}$ & $\begin{array}{l}\text { Nine one-hour } \\
\text { HWC sessions } \\
\text { over 14wk }\end{array}$ & $\mathrm{n} / \mathrm{a}$ & $\begin{array}{l}\text { Patient had a clinically meaning- } \\
\text { ful (11-lb) weight loss main- } \\
\text { tained at two-and-a-half years } \\
\text { and a normal BMI. She also had } \\
\text { decreased cholesterol, LDLs, } \\
\text { and saturated fat with increased } \\
\text { physical activity, fruit intake, } \\
\text { vegetable intake, and HDLs at }\end{array}$ \\
\hline $\begin{array}{l}\text { Kuman- } \\
\text { yika et } \\
\text { al. }{ }^{[47]}\end{array}$ & $\begin{array}{l}\text { RCT; HWC } \\
\text { vs. usual } \\
\text { care }\end{array}$ & $\begin{array}{l}\text { Medical } \\
\text { Assistant } \\
\text { or auxiliary } \\
\text { health pro- } \\
\text { fessional }\end{array}$ & $\begin{array}{l}340 \\
\text { obese } \\
\text { adults }\end{array}$ & $\begin{array}{l}\text { In person, one-on-one } \\
\text { HWC adapted from } \\
\text { Diabetes Prevention } \\
\text { Program \& quarterly } \\
\text { PCP counseling }\end{array}$ & $\begin{array}{l}\text { Six months } \\
\text { of weekly } \\
\text { HWC followed } \\
\text { by HWC } \\
\text { every-oth- } \\
\text { er-month for } \\
\text { six months }\end{array}$ & $\begin{array}{l}\text { Quarter- } \\
\text { ly PCP } \\
\text { counsel- } \\
\text { ing }\end{array}$ & $\begin{array}{l}\text { Mean weight loss in HWC group } \\
\text { over twice as high as control } \\
\text { group at one year }(\mathrm{P}=0.15) \text {, } \\
\text { while clinically meaningful } \\
\text { weight loss was achieved in } \\
10.2 \% \text { of control group and } \\
22.5 \% \text { of HWC group }(\mathrm{P}= \\
0.022)\end{array}$ \\
\hline $\begin{array}{l}\text { Moore et } \\
\text { al. }^{[48]}\end{array}$ & Case Study & $\begin{array}{l}\text { Health } \\
\text { Coach upon } \\
\text { physician } \\
\text { referral }\end{array}$ & $\begin{array}{l}\text { Adult, } \\
\text { obese } \\
\text { female }\end{array}$ & $\begin{array}{l}\text { In person, one-on-one } \\
\text { HWC }\end{array}$ & $\begin{array}{l}14 \text { sessions } \\
\text { over } 11 \text { months }\end{array}$ & $\mathrm{n} / \mathrm{a}$ & $\begin{array}{l}\text { Patient reduced weight by } 21 \% \\
\text { (clinically meaningful), lowering } \\
\text { BMI from class II obese to } \\
\text { overweight. She also achieved } \\
\text { normal blood glucose levels } \\
\text { and reported increased physical } \\
\text { activity, pain reduction, and } \\
\text { improved mobility after HWC }\end{array}$ \\
\hline
\end{tabular}




\begin{tabular}{|c|c|c|c|c|c|c|c|}
\hline $\begin{array}{l}\text { Riggs et } \\
\text { al. }{ }^{[44]}\end{array}$ & $\begin{array}{l}\text { Single } \\
\text { cohort be- } \\
\text { fore-and-af- } \\
\text { ter trial }\end{array}$ & $\begin{array}{l}\text { Mas- } \\
\text { ters-level } \\
\text { training in- } \\
\text { tervention- } \\
\text { ists with } \\
8+\text { years } \\
\text { experience } \\
\text { in behavior } \\
\text { intervention }\end{array}$ & $\begin{array}{l}38 \text { par- } \\
\text { ent-child } \\
\text { pairs of } \\
\text { over- } \\
\text { weight } \\
\text { or obese } \\
\text { children } \\
\text { ages 6-12 } \\
\text { years } \\
\text { with at } \\
\text { least one } \\
\text { over- } \\
\text { weight } \\
\text { parent }\end{array}$ & $\begin{array}{l}\text { Parent-child pair HWC } \\
\text { sessions in-person }\end{array}$ & $\begin{array}{l}\text { 12- to-16 } \\
\text { weekly HWC } \\
\text { sessions } \\
\text { consisting of a } \\
5 \text { - } 10 \text { minute } \\
\text { group session } \\
\text { to discuss goals } \\
\text { followed by } \\
\text { hour-long par- } \\
\text { ent-child pair } \\
\text { HWC sessions } \\
\text { (total } 65 \text { - } 70 \\
\text { minute weekly) }\end{array}$ & $\begin{array}{l}\text { Pre vs. } \\
\text { post } \\
\text { HWC } \\
\text { interven- } \\
\text { tion }\end{array}$ & $\begin{array}{l}\text { Parent-reported child partici- } \\
\text { pants had a significant increase } \\
\text { in quality-of-life score }(\mathrm{P}= \\
0.002) \text {. } \\
\text { Among children who completed } \\
\text { HWC, mean change in } Z \text {-BMI } \\
\text { was a } 0.1 \text { decrease }(\mathrm{P}<0.001) \text {. }\end{array}$ \\
\hline $\begin{array}{l}\text { Schwartz } \\
\text { et al. }{ }^{[31]}\end{array}$ & Case Study & $\begin{array}{l}\text { Registered } \\
\text { Dietician } \\
\text { Nutritionist } \\
\text { Wellness } \\
\text { Coach and } \\
\text { Physician }\end{array}$ & $\begin{array}{l}\text { Adult, } \\
\text { obese } \\
\text { male }\end{array}$ & $\begin{array}{l}\text { In-person, one-on-one } \\
\text { HWC }\end{array}$ & $\begin{array}{l}\text { Monthly HWC } \\
\text { with RDN and } \\
\text { PCP for one } \\
\text { year followed } \\
\text { by one year } \\
\text { of bi-monthly } \\
\text { HWC and one } \\
\text { year quarterly } \\
\text { HWC with } \\
\text { RDN alone } \\
\text { (total three } \\
\text { years) }\end{array}$ & $\mathrm{n} / \mathrm{a}$ & $\begin{array}{l}\text { Patient achieved an initial } \\
\text { clinically meaningful ( } 30-1 b) \\
\text { weight loss in three months to } \\
\text { prepare for bariatric surgery, but } \\
\text { opted to continue HWC instead. } \\
\text { He Patient had and maintained } \\
\text { a } 240-1 b \text { weight loss at three } \\
\text { years and also lowered his blood } \\
\text { glucose levels and cholesterol to } \\
\text { normal. }\end{array}$ \\
\hline $\begin{array}{l}\text { Sherman } \\
\text { et al. }{ }^{[49]}\end{array}$ & Case Study & $\begin{array}{l}\text { Health } \\
\text { Coach }\end{array}$ & $\begin{array}{l}\text { Adult, } \\
\text { obese } \\
\text { male }\end{array}$ & $\begin{array}{l}\text { Three in-person, one- } \\
\text { on-one HWC sessions } \\
\text { and seven telephonic } \\
\text { HWC sessions deliv- } \\
\text { ered over } 12 \text { months }\end{array}$ & $\begin{array}{l}10 \text { HWC } \\
\text { sessions over } \\
\text { a year }\end{array}$ & $\mathrm{n} / \mathrm{a}$ & $\begin{array}{l}\text { Patient had a clinically meaning- } \\
\text { fulweight loss of } 23 \% \text { over one } \\
\text { year, reducing BMI from obese } \\
\left(31 \mathrm{~kg} / \mathrm{m}^{2}\right) \text { to normal }(24 \mathrm{~kg} / \\
\left.\mathrm{m}^{2}\right) \text {, reduced hypertensive blood } \\
\text { pressure to normal, lowered } \\
\text { resting heart rate from } 77 \text { to } 58 \\
\text { bpm. }\end{array}$ \\
\hline $\begin{array}{l}\text { Taveras } \\
\text { et al. }{ }^{[45]}\end{array}$ & RCT & $\begin{array}{l}\text { Trained } \\
\text { Health } \\
\text { Coach }\end{array}$ & $\begin{array}{l}750 \text { Chil- } \\
\text { dren ages } \\
2-12 \text { con- } \\
\text { sidered } \\
\text { over- } \\
\text { weight or } \\
\text { obese }\end{array}$ & $\begin{array}{l}\text { Tailored HWC for } \\
\text { child-parent pairs } \\
\text { according to parent } \\
\text { preferences including: } \\
\text { telephonic, email, mail, } \\
\text { text message, video } \\
\text { visits, and in-person- } \\
\text { HWC. Also given } \\
\text { education material and } \\
\text { community resources } \\
\text { for support. }\end{array}$ & $\begin{array}{l}\text { Six } 20 \text { minute } \\
\text { sessions every } \\
\text { other month for } \\
\text { one year }\end{array}$ & $\begin{array}{l}\text { Enhanced } \\
\text { pediatric } \\
\text { care and } \\
\text { non-tai- } \\
\text { lored } \\
\text { HWC }\end{array}$ & $\begin{array}{l}\text { Lower BMI in children in } \\
\text { intervention group and improved } \\
\text { health-related quality of life by } \\
\text { parents and children. }\end{array}$ \\
\hline $\begin{array}{l}\text { Wadden } \\
\text { et al. }{ }^{[51]}\end{array}$ & $\begin{array}{l}\text { RCT; HWC } \\
\text { intervention } \\
+ \text { weight } \\
\text { loss medica- } \\
\text { tion or meal } \\
\text { replacement } \\
\text { vs. usual } \\
\text { care }\end{array}$ & $\begin{array}{l}\text { Coach- } \\
\text { ing-trained } \\
\text { MAs }\end{array}$ & $\begin{array}{l}390 \\
\text { obese } \\
\text { adults } \\
\text { with at } \\
\text { least two } \\
\text { compo- } \\
\text { nents of } \\
\text { metabol- } \\
\text { ic syn- } \\
\text { drome }\end{array}$ & $\begin{array}{l}\text { Brief LC group re- } \\
\text { ceived } 10 \text { - } 15 \text { minute, } \\
\text { monthly, in-person, } \\
\text { one-on-oneHWC meet- } \\
\text { ings \& quarterly PCP } \\
\text { counseling } \\
\text { Enhanced LC received } \\
10 \text { - } 15 \text { minute, month- } \\
\text { ly, in-person, one-on- } \\
\text { one HWC meetings \& } \\
\text { weight loss medication } \\
\text { OR meal replacement } \\
\& \text { monthly PCP coun- } \\
\text { seling }\end{array}$ & $\begin{array}{l}\text { Monthly } 10-15 \\
\text { minute HWC } \\
\text { sessions for } \\
\text { two years }\end{array}$ & $\begin{array}{l}\text { Quarter- } \\
\text { ly PCP } \\
\text { counsel- } \\
\text { ing to } \\
\text { review } \\
\text { patient } \\
\text { weight } \\
\text { loss \& } \\
\text { discuss } \\
\text { weight } \\
\text { loss hand- } \\
\text { outs, with } \\
\text { no strat- } \\
\text { egies for } \\
\text { behavior } \\
\text { change }\end{array}$ & $\begin{array}{l}\text { Enhanced LC demonstrated } \\
\text { greatest percent weight loss } \\
\text { compared to brief LC }(\mathrm{P}=0.04) \\
\text { and control }(\mathrm{P}<0.001) \text {, while } \\
\text { brief LC group achieved over } \\
\text { twice the weight loss of control } \\
(\mathrm{P}=0.22) \text {. Clinically meaning- } \\
\text { ful weight loss was achieved } \\
\text { in } 21.5 \% \text { of control group, } \\
26.0 \% \text { of brief LC, and } 34.9 \% \\
\text { of enhanced LC at } 24 \text { months ( } \mathrm{P} \\
=0.02)\end{array}$ \\
\hline
\end{tabular}




\begin{tabular}{|l|l|l|l|l|l|l|l|}
\hline $\begin{array}{l}\text { Yang et } \\
\text { al. }{ }^{52]}\end{array}$ & Case Study & $\begin{array}{l}\text { Trained } \\
\text { Wellness } \\
\text { Coach }\end{array}$ & $\begin{array}{l}\text { Adult, } \\
\text { obese } \\
\text { woman }\end{array}$ & $\begin{array}{l}\text { Telephonic, one-on-one } \\
\text { coaching session }\end{array}$ & $\begin{array}{l}\text { Eight bi-week- } \\
\text { ly 30 minute } \\
\text { HWC sessions } \\
\text { for 24 weeks }\end{array}$ & na & $\begin{array}{l}\text { Patient had clinically meaningful } \\
\text { weight loss, decreased BMI, and } \\
\text { reported increased confidence } \\
\text { towards healthier behaviors, } \\
\text { lifestyle, and time management. }\end{array}$ \\
\hline
\end{tabular}

Clinically meaningful weight loss $=>5 \%$ of initial weight within six months

$\mathrm{CV}=$ Cardiovascular, $\mathrm{LC}=$ lifestyle counseling, HIP $=$ Healthy Initiatives Program, $\mathrm{HWC}=$ Health and Wellness Coaching, $\mathrm{RCT}=$ randomized clinical trial.

YLP = Youth-Lifestyle Program

\section{Discussion and Proposal}

Our review of literature shows that health and wellness coaching has been efficacious in weight management and treatment for overweight and obese patients in a clinical setting. Although, the variety of coaching methods for weight management has yielded a mixture of results (Table 1), certain trends have come to light.

First, successful delivery of coaching requires adequate training in health and wellness coaching. For instance, patients receiving coaching from coaching-trained auxiliary health professionals ${ }^{[47,50,54]}$, non-primary care team coaches upon PCP referral ${ }^{[31,49]}$, a physician with lifestyle medicine and wellness coaching training ${ }^{[34]}$, and coaches collaborating with $\mathrm{PCPS}^{[10,51]}$, all resulted in clinically meaningful weight loss. However, weight management with a physician having expertise in nutrition, exercise, or mental health was not necessary to yield significant changes in weight loss ${ }^{[46]}$. This suggests that medical expertise does not necessarily provide health care professionals with the counseling or coaching skills to motivate and empower patients to initiate lifestyle changes for weight loss. This also confirms that the skills learned in coaching are efficacious in prompting lifestyle and behavior change for weight loss. However, many patients relate that they would be more likely to lose weight and engage in weight-loss behavior upon their doctor's recommendation ${ }^{[19,27]}$, making it commendable for a physician or other PCP to integrate coaching skills into their clinical care services for patients with weight problems. This has integrated within the primary care team ${ }^{[3,50,54]}$, upon PCP referral for coaching ${ }^{[9,31]}$, and with PCP plus coach collaboration ${ }^{[10,47,51]}$ towards helping patients achieve clinically meaningful weight loss. Alternatively, physicians interested in personally treating their patients with coaching could complete their training and certification via the International Coaching Federation (www.coachfederation.org), the largest professional coaching credentialing program. Another option is wellcoaches School of Coaching (www.wellcoachesschool.com), which has developed programs and curriculum for evidence-based education, training, and certification in health and wellness coaching. Further, coaching training could be incorporated into the curriculum for Medical School, Residency, or a Fellowship. Particular residencies such as Pediatrics, Family Medicine, Lifestyle Medicine Endocrinology, and Psychiatry may be ideal specialties to include a coaching training program for students. Keeping aligned with this notion, in recent, National Board of Medical Education (NBME) and National Consortium for Credentialing Health \& Wellness Coaches (NCCHWC) have signed an agreement for the launch of a National Certification Examination for Health and Wellness Coaches (NCCHWC) in the US. Similar accommodations and training would also need to be available for other PCPs, such as Physician Assistants and Nurse Practitioners. Perhaps a new specialization or field in Medicine, Health and Wellness Coaching, may need to be realized.

Second, coaching has been successfully integrated into clinical care towards clinically meaningful weight loss in a variety of and settings Clinically meaningful weight loss was achieved in coaching for groups of patients ${ }^{[10]}$, through oneon-one coaching ${ }^{[10,31,34,47-49,51,52,54]}$, and in person ${ }^{[8,10,31,34,47-49,51]}$, Clinically meaningful weight loss also resulted with coaching delivered remotely via telephone ${ }^{[10,49,52]}$ as well as website and email support ${ }^{[10]}$. These findings also indicate that coaching can be tailored to according to patient and provider preferences and convenience. Significant weight-loss has also achieved with a mobile device health coaching application and weekly telephonic coaching ${ }^{[15,53]}$, showing the usefulness of technology-based coaching. Remote coaching combining technology would have merits for patients with busy lifestyles or lacking time to make regular visits to their PCP or coach. These findings suggest coaching can be tailored to suit the preferences and needs of patients and providers depending on predilections and resources for mode of coaching delivery. Study methods which were unsuccessful in achieving either clinically meaningful or statistically significant weight loss included parent-child groups-delivered coaching at a low-intensity dose ${ }^{[4,45]}$, coaching delivered by providers lacking coaching training ${ }^{[46]}$, and a short-term eightweek coaching session delivered by a $\operatorname{coach}^{[52]}$. This reiterates the importance of coaching with a professional who has completed training and certification, as discussed earlier. Additionally, these results indicate that although the method of coaching can be tailored to suit patients, the dose required for successful weight loss with coaching is much more rigid, as discussed below.

Third, in regards to dosage, another way to increase the efficacy of coaching is by offering coaching at moderate to high-intensity frequency. Obese and overweight patients participating in coaching at a moderate to high-intensity are more likely to sustain clinically meaningful weight loss that will improve their health outcomes ${ }^{[10,31,3447-49,51,54]}$. Longer time frames for coaching were also correlated with higher likelihood of maintenance of clinically meaningful weight loss at follow up. Moderate - to high-intensity coaching sessions offered either in person or over the phone for 4 - 5 months resulted in positive improvements in weight status, but these results were short-term lacking significance at follow-up ${ }^{[2,54]}$. Instead, moderate- to high-intensity coaching offered for at least a year, helped more obese and overweight patients achieve and sustain clinically meaningful weight loss years later ${ }^{[1,31,48,49,51]}$. Interestingly though, one fourmonth, high-intensity coaching intervention resulted in clinically meaningful weight loss maintained at follow-up two-anda-half years later ${ }^{[34]}$. This raises the important concept that the 
duration of coaching intervention may depend on both the stage of change the patient is in at intervention onset as well as the extent of weight loss the patient needs to achieve. Importantly, although coaching for short durations may facilitate weight loss, if these changes are not maintained patients are less likely to achieve improvement in obesity-related sequel, making longer durations of treatment optimal to ensure maintenance of weight loss.

Fourth, concerning patient safety, coaching may lack the side effects associated with the pharmacological management of weight loss. Most studies neglected to report on adverse events, while two studies reported no adverse side effects resulting from coaching ${ }^{[47,54]}$. However, one study did report a patient was assaulted while exercising as part of their coaching process $^{[10]}$. More research may need to be done to investigate the safety and risk of adverse events with coaching. Alternatively, the increased risk of adverse events with weight loss drugs is well known. The risk of gastrointestinal events with Orlistat ${ }^{[55]}$ and side effects of headache, nausea, and dizziness with Lorcaserin $^{[56]}$, are well-documented. The decreased risk of adverse events in combination with the documented benefits of coaching makes it a safe and effective first-line therapy for behavior and lifestyle modification towards weight loss. This is particularly true of patients in which pharmacological therapy or bariatric surgery for weight loss is contraindicated or not recommended. Though beyond the scope of this review, coaching and pharmacotherapy have been shown to be more effective than either therapy alone in the care and management of weight loss for obese patients $^{[15,51]}$. However, in one study, patients receiving coaching without pharmacotherapy still achieved double the weight loss of usual care patients ${ }^{[51]}$.

Fifth, one limitation we noticed through the present review is of the extensive time demands necessary for health and wellness coaching process in clinic care. The coordination of incorporating health and wellness coaching into clinical care may make it a difficult role for a PCP. In reality, there are limited contact hours between a PCP and patient, and PCPs must often triage acute diseases ahead of chronic conditions when caring for patients. However, the time constraints that may prevent physicians from utilizing coaching directly may be circumvented several ways. Studies have shown auxiliary health professionals with coaching training working under PCP care and management recommendations have been effective in helping patients achieve clinically meaningful weight $\operatorname{loss}^{[31,47,51,54]}$. Similar significant decreases in weight have been reported with non-medical personnel coaches and PCP collaboration ${ }^{[10,48,49,52]}$. A PCP may also remain a part of their patient's coaching process similar to referring a patient to a physician specialist. PCPs could recommend patients who are obese or overweight to a health and wellness coach. This would allow the patient's PCP and coach to collaborate in approaching the patient's coaching plan and integrate their patient's weight management into a holistic picture of their overall health.

Sixth, the logistics of the coaching process is another component to consider in integrating coaching into clinical care to maximize efficacy of weight management. PCPs may need to contract out coaches or have auxiliary health professionals with coaching training working at their facilities due to insufficient time to incorporating coaching personally into the care of their patients. Other difficulties include how insurance com- panies would code, charge, and cover the cost of a prolonged treatment with coaching or if patients would be responsible for these payments out of pocket, as obesity is not considered a billable diagnosis for many insurance companies ${ }^{[54]}$. Only one study documented cost of treatment, and the patient paid $\$ 700$ out of pocket for her 11 months of high-intensity coaching, for which she was referred ${ }^{[48]}$. Alternatively, one cost-effective analysis showed projected annual costs for Orlistat was $\$ 1,518$, while Lorcaserin was $\$ 1,743^{[57]}$, suggesting coaching is likely the more affordable option in addition to having a decreased risk of adverse reactions.

Lastly, health and wellness coaching can facilitate and sustain prolonged weight loss, increasing many positive health outcomes. In terms of efficacy in long-term weight management, our present review of coaching intervention studies have shown that subjects were able to lose and maintain clinically meaningful weight loss with concurrent coaching for one year ${ }^{[49,50]}$, up to two years ${ }^{[10,51]}$, and even up to three years ${ }^{[31]}$. One patient in particular was able to maintain clinically meaningful weight loss over two-and-a-half years post-coaching, citing physician emails post-treatment as reminders for her to continue to engage in her active lifestyle ${ }^{[34]}$. Additionally, coaching resulted in improvements in other health measurements such as $\mathrm{HbAlC}$, blood pressure, triglycerides, cholesterol, and more. These findings indicate coaching is efficacious for treating and preventing other co-morbidities associated with obesity and chronic diseases such as diabetes, cardiovascular disease for prolonged, sustainable periods. However, this also highlights the need for additional investigations as to the effectiveness of coaching and maintenance of weight loss invariable. It would be helpful to examine if five, ten, or twenty years after the coaching process has formally ended if patients were able to maintain their healthier lifestyles and habits, and adapt what they learned in the coaching process towards improving their health and longevity.

Overall, health and wellness coaching, albeit a time consuming process, has important and promising implications in clinical practice. Coaching helps children, adolescents, and adults who are overweight or obese improve their lifestyles and health through weight loss as well as improving factors related to diabetes and cardiovascular disease. Other improvements resulting from coaching include increased physical activity, improved quality of life, improved time management, and increased self-efficacy for healthy lifestyle choices. Further the physiological, psychological, and social consequences associated with obesity can improve through coaching for weight loss. The relationship between a trained coach and patient also enables for personalize care to best suit patient needs and preferences. The approach of patient-care and specific goals set by patients can be tailored to best meet the needs of the patient, which increases the chances of successful weight loss and lifestyle changes within personally identified desired domains.

\section{Conclusions}

The obesity pandemic needs to be tackled in an efficacious way to ensure meaningful, sustainable weight loss in individuals whom their health and quality of life are gravely threatened by their weight. While physicians face the challenge of such health issue present among their patients, a safe, efficacious, and potentially cost-effective means of addressing 
overweight and obesity problems may be possible through integrating health and wellness coaching into a clinical care service. The can be best accomplished via health and wellness coaching towards healthier lifestyles and behavior modification for weight management in a clincial setting. Health and wellness coaching is best integrated for a prolonged period of time, via various modes of delivery, and with a coach who has completed coaching training. Coaching may be a useful adjunct in a primary care setting for obese and overweight patients as a highly efficacious approach to warrant success of weight management. However, concerning the long, committed coaching process necessary for sustainable weight management outcomes on the client end, the benefit of incorporating coaching for a clinical care service must be weighed against the time required for providers to integrate coaching process into the clinical setting. Alternatively, this challenge can be resolved by having PCPs and other staff be trained in coaching or team up with trained coaches.

\section{Strengths and Limitations}

Our paper can contribute to the growing evidence of health and wellness coaching as an efficacious approach to addressing weight issues among patients in a clinic setting by offering an extensive review of the related references for the past ten years. Also, our review provides in-depth knowledge and successful methods as to how coaching has been efficacious in helping patients improve their weight-related health long term. In particular, the findings in the present study can contribute to the need of filling in the gap of PCCs' roles to serve the patients with overweight or obese weight issues by suggesting a way to incorporate coaching into the clinical care of patients who are obese or overweight. However, the limitation of this review exist by excluding pharmacological interventions in obesity and overweight management, which sometimes can be vital to the successful care of the overweight or obese patient. There are also limitations in the studies reviewed that investigate coaching in the primary care setting for only for weight loss and the management of overweight and obesity.

\section{Future Direction}

It may be valuable to conduct a survey study among the primary care providers in the local community to investigate the perception, approach, process, and outcome of health and wellness coaching integrated into the clinical care of overweight and obese patients. This could be done in collaboration with JPS Health Care System and University of North Texas Health Science Center medical system, affiliated with the authors' medical school. Further, this would allow the authors to implement a coaching training program for providers interested in incorporating health and wellness coaching into their practice for overweight or obese patients. As the leading author is a lifestyle interventionist and a certified health and wellness coach from the well coaches School, the training program for clinical staff (e.g. physicians, nurses, physician/medical assistant, clinical psychologist, registry dietician, exercise physiologist or fitness expert)addressing the weight issues in the clinic can be easily set up and implemented. The training program in turn would provide the authors with an opportunity to collaborate with the healthcare providers trained in health and wellness coaching to conduct a coaching-based weight management intervention program or offer a coaching-embedded clinical care service in improving health outcomes for their patients with overweight and obesity issues.

\section{References}

1. Padula, W.V., Allen, R.R., Nair, K.V. Determining the cost of obesity and its common comorbidities from a commercial claims database. (2013) Clinical Obesity 4(1): 53-58.

2. Allen, J.K., Stephens, J., Himmerlfarb, C.R.D., et al. Randomized Controlled Pilot Study Testing Use of Smartphone Technology for Obesity Treatment. (2013) Journal of Obesity 2013(2013):1- 77.

3. Brown, C.L., Halvorson, E.E., Cohen, G.M., et al. Addressing Childhood Obesity, Opportunities for Prevention. (2015) Pediatr Clin North Am 62(5):1241-1261.

4. Carvajal, R., Wadden, T.A., Tsai, A.G., et al. Managing obesity in primary care practice: a narrative review. (2013) Ann N Y Acad Sci 1281:191-206.

5. Fan, M., Jin, Y., Khubchandani, J. Overweight Misperception among Adolescents in the United States. (2014) J Pediatr Nurs 29(6): 536-546. 6. Flegal, K.M., Carroll, M.D., Kit, B.K., et al. Prevalence of obesity and trends in the distribution of body mass index among US adults, 1999-2010. (2012) J Americ Med Asso 307(5): 491-497.

7. Greiner, K.A., Born, W., Hall, S., et al. Discussing Weight with Obese Primary Care Patients: Physician and Patient Perceptions. (2008) J Gen Intern Med 23(5): 581-587.

8. U.S. Preventive Services Task Force, Moyer, V.A. Screening for and Management of Obesity in Adults: U.S. Prevention Services Task Force Recommendation Statement. (2012) Ann Intern Med 157(5): 373-378.

9. Perrin, E.M., Finkle, J.P., Benjamin, J.T. Obesity prevention and the primary care pediatrician's office. (2007) Curr Opin Pediatr 19(3): 354361.

10. Appel, L.J., Clark, J.M., Yeh, H., et al. Comparative Effectiveness of Weight-Loss Interventions in Clinical Practice. (2011) NEJM 365(21): 1959-1968.

11. Glauser, T.A., Roepke, N., Stevenin, B., et al. Physician knowledge about and perceptions of obesity management. (2015) Obesity Research \& Clinical Practice 9(6): 573-583.

12. Mc.Kinney, Skolnik, N., Chrusch, A., et al. Diagnosis and Management of Obesity. (2013) American Academy of Family Physicians 1-26. 13. Nguyen, D.M., El-Serag, H.B. The Epidemiology of Obesity. (2010) Gastroenterol Clin North Am 39(1): 1-7.

14. Tao, M., Rangarajan, K., Paustian, M.L., et al. Dialing In: Effect of Telephonic Wellness Coaching on Weight Loss. (2014) Am J Manag Care 20(2): e35-42.

15. Tucker, L.A., Cook, A.J., Nokes, N.R., et al. Telephone-Based Diet and Exercise Coaching and a Weight-loss Supplement Result in Weight and Fat Loss in 120 Men and Women. (2008) Am J Health Promot 23(2): 121-129.

16. Han, J.C., Lawlor, D.A., Kimm, S.Y.S. Childhood obesity. (2010) Lancet 375(9727): 1737-1748.

17. Bramlage, P., Wittchen, H.U., Pittrow, D., et al. Recognition and management of overweight and obesity in primary care in Germany. (2004) Int J Obes 28(10): 1299-1308.

18. Phelan, S., Nallari, M., Darroch, F.E., et al. What Do Physicians Recommend To Their Overweight and Obese Patients? (2009) Jour Am Board of Fam Med 22(2): 115-122.

19. Berning, J. The role of physicians in promoting weight loss. (2015) Econ Hum Bio104-115.

20. Clark, M.M., Bradley, K.L., Jenkins, S.M., et al. The Effectiveness of Wellness Coaching for Improving Quality of Life. (2014) Mayo Clin Proc 89(11): 1537-1544.

21. Gurnani, M., Birken, C., Hamilton, J. Childhood Obesity Causes, Consequences, and Mangement. (2015) Pediatri Clin North Am 62(4): 821-840. 
22. Daniels, S.R., Hassink, S.G. The Role of the Pediatrician in Primary Prevention of Obesity. (2015) Pediatrics 136(1): e275-292.

23. Li, Q., Blume, S.W., Huang, J.C., et al. Prevalence and healthcare costs of obesity-related comorbidities: evidence from an electronic medical records system in the United States. (2015) J Med Econ 18(12): 1020-1028.

24. Ng, M., Fleming, T., Robinson, M., et al. Global, regional and national prevalence of overweight and obesity in children and adults 1980-2013: A systematic analysis for the Global Burden of Disease Study 2013. (2014) Lancet 384(9945): 766-781.

25. Jensen MD, Ryan DH, Donato KA, et al. Executive summary: Guidelines (2013) for the management of overweight and obesity in adults: a report of the American College of Cardiology/American Heart Association Task Force on Practice Guidelines and the Obesity Society published by the Obesity Society and American College of Cardiology/ American Heart Association Task Force on Practice Guidelines. Based on a systematic review from the Obesity Expert Panel, 2013. (2014) Obesity (Silver Spring). 22(25).

26. Spear, B.A., Barlow, S.E., Ervin, C., et al. Recommendations for treatment of child and adolescent overweight and obesity. (2007) Pediatrics 120(4).

27. Bleich, S.N., Pickett-Blakely, O., Cooper, L.A. Physician practice patterns of obesity diagnosis and weight-related counseling. (2011) Patient Educ Couns 82(1): 123-129.

28. Aschbrenner, K.A., Naslund, J.A., Barre, L.K., et al. Peer Health Coaching for overweight and obese individuals with serious mental health illness: intervention development and initial feasibility study. (2015) Transl Behav Med 5(3): 277-284

29. Wolever, R.Q., Simmons, L.A., Sforzo, G.A., et al. A Systematic Review of the Literature on Health and Wellness Coaching: Defining a Key Behavioral Intervention in Healthcare. (2013) Global Adv Health Med 2(4): 38-57.

30. Turner, K.M., Salisbury, C., Shield, J.P. Parents' views and experiences of childhood obesity management in primary care: a qualitative study. (2012) Fam Pract 29(4): 476-481.

31. Schwartz, J. Wellness Coaching for Obesity: A Case Report. (2013) Glob Adv Health Med 2(4): 68-70.

32. Spurrier, N.J., Magarey, A., Wong, C. Recognition and management of childhood overweight and obesity by clinicians. (2006) J Paediatr Child Health 43(7-8): 411-418.

33. Centers for Disease Control and Prevention. Summary health statistics for U.S. adults: National Health Interview Survey, 2010. Hyattsville, MD: National Center for Health Statistics. (2012)Vital and Health Statistics 10 (252): 1-217.

34. Frates, E.P., Crane, M.E. Lifestyle medicine consulting walking meetings for sustained weight loss. (2016) BMJ Case Rep 1-2.

35. Simmons, L.A., Wolever, R.Q. Integrative Health Coaching and Motivational Interviewing: Synergistic Approaches to Behavior Change in Healthcare. (2013) Glob Adv Health Med 2(4): 28-35.

36. Caldwell, K.L., Gray, J., Wolever, R.Q. The Process of Patient Empowerment in Integrative Health Coaching: How Does It Happen? (2013)Glob Adv Health Med 2(3): 48-57

37. Frates, E.P., Moore, M.A., Lopez, C.N., et al. coaching for behaviors change in physiatry. (2011) Am J Phys Med Rehabil 90(12): 10741082.

38. Wadden, T.A., Butryn, M.L., Hong, P.S., et al. Behavioral Treatment of Obesity in Patients Encountered in Primary Care Settings A Systematic Review. (2014) JAMA 312(17): 1779-1791.

39. Gazelle, G. Webinar: Tackling Burnout in Physicians: How Coaching Can Help.(2016) Instituteofcoaching.org [Cited 13 July 2016].

Journal Title: Journal of Diabetes and Obesity (JDO)

Journal Short Name: J diabetes Obes
40. Annesi, J.J., Tennant, G., Chapman, A. et al. Effects of the Coach Approach intervention of psychosocial predictors of exercise and subsequent changes in glucose metabolism, cardio respiratory functioning, and body composition: a pilot project of the interior health of British Columbia and a local YMCA. (2012) Archive Exerci Health Disea 3(12): 162 .

41. Sharma, A.E., Willard-Grace, R., Hessler, D., et al. What happens after health coaching? Observational study 1 year following a randomized controlled trial. (2016) Annals of Family Medicine 4(3): 200-207. 42. Kivelä, K., Elo, S., Kyngäs, H., et al. The effects of health coaching on adult patients with chronic disease: A systemic review. (2014) $\mathrm{Pa}$ tient Educ Couns 97(2): 147-157.

43. Wayne. Health coaching reduces HbA1c in Type 2 Diabetic Patients from a Lower-Socioeconomic Status Community: A Randomized Controlled Trial. (2015) J Med Internet Res 17(10): e224.

44. Riggs, K.R., Lozano, P., Mohelnitzky, A., et al. An Adaption of Family-Based Behavioral Obesity Treatments for a Primary Care Setting: Group Health Family Wellness Program Pilot. (2014) Perm J 18(3): 4-10.

45. Taveras, E.M., Marshal, R., Shirif, M.,et al. Connect for Health: Design of a Clinical-Community Childhood Obesity Interventions Testing Best Practices of Positive Outliers. (2015) Contemp Clin Trials 45: 287-295.

46. Ball, G.D.C., Mackenzie-Rife, K.A., Newton, M.S., et al. One-onone lifestyle coaching for managing adolescent obesity: Findings from a pilot, randomized controlled trial in a real-world, clinical setting. (2007) Paediatr Child Health 16(6): 345-350.

47. Kumanyika, S.K., Fassbender, J.E., Sarwer, D.B., et al. One-Year Results of Think Health! Study of Weight Management in Primary Care Practices. (2012) Obesity 20(6): 1249-1257.

48. Moore, C. Case Report of Hemoglobin A1c and Weight Reduction in Integrative Health Coaching. (2013) Glob Adv Health Med 2(3): 8789.

49. Sherman, R.P., Crocker, B., Dill, D., et al. Health Coaching Integration into Primary Care for the Treatment of Obesity. (2013) Glob Adv Health Med 2(4): 58-60.

50. terBogt, N.C.W., Bemelmans, W.J.E., Beltman, F.W., et al. Preventing Weight Gain One-Year Results of a Randomized Lifestyle Intervention. (2009) Am J Prev Med 37(4): 270-277.

51. Wadden, T.A., Volger, S., Sarwer, D.B., et al. A Two-year Randomized Trial of Obesity Treatment in Primary Care Practice. (2011) NEJM 365(21): 1969-1979.

52. Yang, N.Y., Wroth, S., Parham, C., et al. Personalized Health Planning With Integrative Health Coaching to Reduce Obesity Risk Among Women Gaining Excess Weight During Pregnancy. (2013) Glob Adv Health Med 2(4): 72-77.

53. Thiese, M.S., Effiong, A.c., Ott, U., et al. Clinical Trial on Weight Loss among Truck Drivers. (2015) Int J Occup Environ Med 6: 104112.

54. Tsai, A.G., Wadden, T.A., Rogers, M.A., et al. A primary care intervention for weight loss: results of a randomized controlled pilot study. (2010) Obesity (Silver Spring) 18(8): 1614-1619.

55. Heymsfield, S.B., Segal, K.R., Hauptman, J., et al. Effects of weight loss with orlistat on glucose tolerance and progression to type 2 Diabetes in obese adults. (2000) Arch Intern Med 160(9): 1321-1326.

56. Fidler, M.C., Sanchez, M., Raether, B., et al. A One-Year Randomized Trial of Lorcaserin for Weight Loss in Obese and Overweight Adults: The BLOSSOM Trial. (2011) J Clin Endocrinol Metab 96(10): 3067-3077.

57. Finkelstein, E.A., Kruger, E. Meta- and Cost-Effectiveness Analysis of Commercial weight loss strategies. (2014) Obesity (Silver Spring) 22(9): 1942-1951. 